\title{
MATERNAL AND CHILDHOOD UNDERNUTRITION IN BANGLADESH: KEEPING THE ISSUE ON THE AGENDA
}

Childhood and maternal undernutrition remain as two of the main public health challenges of the 21st century, particularly in low and middle income countries. ${ }^{1}$ Recent estimates suggest that in developing countries 314 (296 to 331) million children younger than 5 years are stunted and 258 (240 to 274 ) million are underweight. ${ }^{2}$ The burden of undernutrition chronically afflicts 171 million children worldwide; India, Bangladesh and Pakistan, account for almost half the world's total underweight children, even though the countries are home to just $29 \%$ of the developing world's under five year old population. ${ }^{3}$ Undernutrition greatly impedes countries' socio-economic development and the potential to reduce poverty. Many of the Millennium Development Goals (MDGs), particularly MDG-1, to eradicate extreme poverty and hunger, and MDG-4, to reduce child mortality, will not be reached unless national development programmes and strategies give priority to the nutrition of women and children. In Bangladesh steady declines have been noted in fertility, maternal, infant and child mortalities. However, the nutritional status of children continues to remain poor and is no longer on track to achieve the MDG-1 target on child underweight rates of $33 \%$ by $2015 .{ }^{4}$

Recent analysis of a total of 8,858 under-two children of the data of National Nutrition Programme baseline survey revealed that $40.5 \%$ of the children were stunted ( $15.6 \%$ severely stunted), $35.4 \%$ were underweight (11.5\% severely underweight) and $17.8 \%$ were wasted (3\% severely wasted). ${ }^{5}$ Analysis of the Bangladesh Demographic Health Survey (BDHS) data showed that there has been some improvement in child nutritional status over the past years. The level of stunting has declined from 51\% in 2004 and $43 \%$ in 2007 to $41 \%$ of children under five in 2011. The pattern and change in wasting has been small and inconsistent. It increased from $15 \%$ in 2004 to $17 \%$ in 2007 , and declined to $16 \%$ by 2011 . The level of underweight has been declining from $43 \%$ in 2004 , to $41 \%$ in 2007 , and to $36 \%$ in $2011 .{ }^{6}$ Although there were modest improvements in past decades, the nutritional status of women in Bangladesh is still alarming. The FSNSP data reported that around $28 \%$ of Bangladeshi women, having under five children in their families, suffered from chronic energy deficiency (CED). ${ }^{7}$ Ahmed et al. ${ }^{5}$ reported that the nutritional status of women in Bangladesh is showing an improving trend. In 1997, $52 \%$ of women had CED; since then, a sustained reduction has been observed in the prevalence of CED; the prevalence being $30 \%$ in 2007 . BDHS data analysis likewise showed that $39.2 \%$ of the mothers of under five children were suffering from CED, while 5.6\% of them had a BMI less than $16 \mathrm{~kg} / \mathrm{m}^{2}{ }^{6}{ }^{6}$

Despite high-level commitment in the context of the MDGs and other initiatives, most developing countries, including Bangladesh are likely to fail in achieving their nutrition-related goals. Recent economic recession and the marked increase in price of rice and other staples over the last years placed Bangladesh in the bottom $25 \%$ of the Global Hunger Index ranking; the nationally representative household food security and nutrition survey conducted in 2008-2009 reported strong linkages between nutritional status and food security indicators related to food price hikes. ${ }^{8}$ Bhutta et al. ${ }^{9}$ showed concern that recent food and economic crises, if unaddressed, could have a significant impact on health and nutrition outcomes in the short term and estimated that childhood stunting rates would increase by $3-7 \%$ and those for wasting by $8-16 \%$, at least in East Asia and the Pacific. The current slow-down or even worsening in the mean Z-scores in Bangladesh echoed their concern.

The multifaceted nature of undernutrition means that it may be effectively addressed only when several sectors and strategic efforts are combined together. Ahmed et al. ${ }^{5}$ recommended that the interventions targeting undernutrition should be scaled up to cover at least $70 \%$ of the total population to show tangible outcomes. Community level integrated packages to address hunger and undernutrition in women and children are being implemented across many countries, this consisted mainly of developing cross-sectoral interventions addressing malnutrition and implementing them in targeted areas/vulnerable communities. The main activities included: (1) Growth Monitoring and Promotion (GMP); (2) Intense nutrition, health, and 
hygiene advocacy; (3) Behaviour Change Communication (BCC) to promote Infant and Young Child Feeding (IYCF); (4) Improving health and immunization services for women and children; (5) Micronutrient and food supplementation; and (6) Expanding treatment and rehabilitation of severely malnourished children both at community and facility levels. The integrated packages gave equal emphasis to preventive (nutrition and health education), and curative (nutrition rehabilitation centers) strategies and implemented a mix of direct and indirect interventions.

The Standing Committee on Nutrition made some recommendations to respond to the food price crisis in reducing the negative effects of the global financial and economic crisis on nutrition of the vulnerable groups. ${ }^{10}$ They suggested that governments should invest in programmes that increase the productivity of small holders, strengthen the livelihoods of the poorest households, and supply local markets throughout the year with affordable, safe and easy to use foods needed for a healthy diet. These need to be complemented with social protection programmes, increased public employment and reinforcement of basic nutrition and health services. In order for development efforts to be successful, it is essential to have the participation of the civil society, farmer's organisations and private sectors at every stage.

\author{
Dr Masuda Mohsena \\ Associate Professor \\ Department of Community Medicine \\ Ibrahim Medical College
}

\section{References}

1. Victora CG, de Onis M, Hallal PC et al. Worldwide timing of growth faltering: revisiting implications for interventions. Pediatrics 2010; 125: e473-80. Epub 2010/02/17.

2. Stevens GA, Finucane MM, Paciorek CJ et al. Trends in mild, moderate, and severe stunting and underweight, and progress towards MDG 1 in 141 developing countries: a systematic analysis of population representative data. The Lancet 2012; 380: 824-34.

3. UNICEF. Monitoring the situation of women and children 2008.

4. World Bank. To the MDGs and Beyond: Accountability And Institutional Innovation In Bangladesh. Dhaka: The World Bank 2007.

5. Ahmed T, Mahfuz M, Ireen S et al. Nutrition of children and women in Bangladesh: trends and directions for the future. Journal of health, population, and nutrition 2012; 30: 1-11. Epub 2012/04/25.

6. BDHS. Bangladesh Demographic and Health Survey 2011. Dhaka, Bangladesh and Calverton, Maryland, USA: National Institute of Population Research and Training, Mitra and Associates, and Macro International 2011.

7. HKI. The Food Security and Nutrition Surveillance Project (FSNSP), Round 4, February to May 2011. 2011 July. Report No: 2.

8. ICDDR B. An overview of under-nutrition in Bangladesh. Health and Science Bulletin 2011; 9: 9-16.

9. Bhutta Z, Baswany F, Feroze A et al. The impact of the food and economic crisis on child health and nutrition. UNICEF Conference, East Asia and the Pacific Islands; 6-7 January; Singapore: UNICEF; 2009.

10. SCN. Global financial and economic crisis - the most vulnerable are at increased risk of hunger and malnutrition. 2009; Available from: http:// www.unscn.org/en/publications/nutrition briefs/ \#Nutrition impacts of global food and financial crises. 